\section{Randomization without replacement using replacement without losing your place}

\section{T. H. NILSSON}

University of Alberta, Edmonton, Alberta, Canada T6G $2 E 9$

Lehman (1977) describes a procedure for sampling without replacement from an array of $\mathrm{N}$ variables that does not require reshuffling the entire array $\mathrm{N}$ times. I have been using a different sampling-withoutreplacement procedure that avoids such reshuffling and also avoids knowing the number of samples remaining. The procedure may be more efficient for on-line applications, since it does not wait upon a randomization routine each time a value is requested. The procedure also facilitates storing data nonrandomly and handling replications.

Start with a one-dimensional array, $X_{1-\mathrm{N}}$, of the various values to be selected. (If $R$ replications are wanted, repeat each value $R$ times in the array and set $N=N \times R$.) For $I=1-N$, select a random number between 1 and $\mathrm{N}$ then interchange the value of the Ith element with the value of the element in the position of the random number. The result is an array of the original values replaced in random order. With this procedure, one randomizes the array beforehand and then takes values from the array in succession. The randomized values are immediately available.

The procedure can be written in a single program line using either FOCAL-RT (Siegel, 1972):

FOR $I=I, N$; SET A $=X(I) ; \operatorname{SET} B=F R A N(N)$; SET $X(I)=X(B)$; SET $X(B)=A$ or BASIC:

FOR I $=\mathrm{I}$ TON $/ \mathrm{A}=\mathrm{X}(\mathrm{I}) / \mathrm{B}=\mathrm{RAN}(\mathrm{N}) / \mathrm{X}(\mathrm{I})=\mathrm{X}(\mathrm{B}) / \mathrm{X}(\mathrm{B})=\mathrm{A} / \mathrm{NEXTN}$

Keeping track of data can be another problem during on-line experiments involving randomized conditions. Once the value of a condition has been selected and used, one must score the data so that it can be retrieved in accord with that condition. A simple solution is possible using an array with a random order of elements. Instead of having the array elements equal the values to be randomized, as above, use the elements as pointers to a second array, $Y_{N}$, which contains the actual condition values. The pointer array, $X_{1}=1, X_{2}=2, \ldots, X_{N}=N$, is first put in random order. Elements are then taken in succession from the pointer array to select values from $\mathrm{Y}_{\mathrm{N}}$ randomly. Upon acquisition of data for a given condition, the same pointer element can direct data storage into a third array at the same position as the selected condition value of $\mathrm{Y}_{\mathrm{N}}$ without having to sort on the basis of the value used.

Randomized pointer arrays are also convenient for handling a variable number of replications. Pointer arrays are easily customized each time the program is used. The array for conditions need not be changed with the following procedure. For $\mathrm{R}$ replications of $\mathrm{N}$ conditions, create a pointer array with $\mathrm{N} \times \mathrm{R}$ elements: $X_{1}=R, X_{2}=R+1, \ldots, X_{N \times R}=R+(N \times R)$ After randomization of the order of the elements, selection of the Ith condition value is accomplished by taking the integer value of $\left(X_{I} \div R\right)$. That integer points to the correct value in $\mathrm{Y}_{\mathrm{N}}$ regardless of replication. Storage of data would be directly according to $\mathrm{X}_{\mathrm{I}}$ into a third array $\mathrm{R}$ times larger than the conditions array.

\section{REFERENCES}

LeHMAN, R. S. An improved BASIC program to produce random samples without replacement. Behavior Research Methods \& Instrumentation, 1977, 9, 555-556.

SIEgEL, W. Comgining FOCAL and assembly language. Behavior Research Methods \& Instrumentation, 1972, 4 105-106.

(Accepted for publication March 15, 1978.) 